\title{
Suspended superconducting weak links from aerosol-synthesized single-walled carbon nanotubes
}

\author{
Jukka-Pekka Kaikkonen ${ }^{1}(\varangle)$, Abhilash Thanniyil Sebastian ${ }^{1}$, Patrik Laiho ${ }^{3}$, Nan Wei ${ }^{3}$, Marco Will ${ }^{1,2}$, Yongping Liao ${ }^{3}$, \\ Esko I. Kauppinen ${ }^{3}$, and Pertti J. Hakonen ${ }^{1,2}(\bowtie)$ \\ ${ }^{1}$ Low Temperature Laboratory, Department of Applied Physics, School of Science, Aalto University, PO Box 15100, FI-00076 Aalto, Finland \\ ${ }^{2}$ QTF Centre of Excellence, Department of Applied Physics, School of Science, Aalto University, P.O. Box 15100, FI-00076 Aalto, Finland \\ ${ }^{3}$ Department of Applied Physics, School of Science, Aalto University, P.O. Box 11100, FI-00076 Aalto, Finland
}

(C) The Author(s) 2020, corrected publication 2020

Received: 16 January 2020 / Revised: 4 August 2020 / Accepted: 4 August 2020

\begin{abstract}
We report a new scheme for fabrication of clean, suspended superconducting weak links from pristine single-walled carbon nanotubes (SWCNT). The SWCNTs were grown using the floating-catalyst chemical vapour deposition (FC-CVD) and directly deposited on top of prefabricated superconducting molybdenum-rhenium (MoRe) electrodes by thermophoresis at nearly ambient conditions. Transparent contacts to SWCNTs were obtained by vacuum-annealing the devices at $900^{\circ} \mathrm{C}$, which enabled proximityinduced supercurrents up to $53 \mathrm{nA}$. SWCNT weak links fabricated on MoRe/palladium bilayer sustained supercurrents up to $0.4 \mathrm{nA}$ after annealing at relatively low temperature of $220{ }^{\circ} \mathrm{C}$. The fabrication process does neither expose SWCNTs to lithographic chemicals, nor the contact electrodes to the harsh conditions of in situ CVD growth. Our scheme facilitates new experimental possibilities for hybrid superconducting devices.
\end{abstract}

\section{KEYWORDS}

carbon nanotube, Josephson junction, floating catalyst chemical vapour deposition, thermophoresis, electrical transport

\section{Introduction}

Pristine single-walled carbon nanotube (SWCNT) quantum dot (QD) devices provide a tunable condensed-matter system that has enabled several new observations in electrical quantum transport [1] and basic interactions of single electrons with mechanical degrees of freedom [2-5]. These devices have also been shown to make very sensitive detectors of mass [6], charge [7], force [8], and magnetic field [9]. Most of the recent advances in carbon nanotube based devices have been obtained using "ultra-clean" suspended SWCNTs fabricated in such a way that the nanotube is not exposed to an electron beam or microfabrication chemicals. In these fabrication schemes, the nanotube is suspended over prefabricated electrodes in the last step of fabrication. This is done either by growing the SWCNTs directly on prefabricated electrodes [10] or by growing the SWCNTs on a separate chip and mechanically transferring them onto the electrodes [11-16]. Fabrication of suspended ultra-clean SWCNT devices of this kind has mostly been targeting single electron devices, whereas experimental studies of high-quality, superconducting SWCNT weak links have remained scarce $[17,18]$. Such hybrid superconductorsuspended SWCNT QD devices could be used to realize several theoretical proposals, including the modification of Josephson currents due to spin-orbit interactions [19], single molecular magnet detection using a SWCNT-superconducting quantum interference device (SWCNT-SQUID) [20-22], detection of Cooper pair entanglement [23-25], and studying the interplay of mechanical vibrations and the Josephson junction dynamics [26, 27]. Furthermore, combined with a microwave cavity, SWCNT-based Josephson inductance [28] offers exciting possibilities in optomechanics [29].

In this paper we report a new scheme for fabrication of suspended ultra-clean SWCNT weak links. Our approach is based on the floating catalyst chemical vapour deposition (FC-CVD) growth of SWCNTs and direct deposition of pristine SWCNTs on prefabricated metallic electrodes at nearambient temperature [30,31]. To obtain good electrical contacts between these aerosol-synthesized SWCNTs and the electrodes, our devices are vacuum annealed at the last step of the fabrication process. SWCNT weak links were fabricated on molybdenum-rhenium (MoRe) alloy and on MoRe/palladium (Pd) bilayer. Annealing temperatures used for MoRe and $\mathrm{MoRe} / \mathrm{Pd}$ contacts were 900 and $220^{\circ} \mathrm{C}$, respectively. Low temperature measurements confirmed the presence of proximityinduced supercurrents in these SNS junctions (superconductornormal conductor-superconductor weak link). The largest measured supercurrent with MoRe contacts was above $50 \mathrm{nA}$ while devices with MoRe/Pd contacts carried supercurrents of about $350 \mathrm{pA}$.

Our results demonstrate that SWCNT weak links can be created even when SWCNTs are deposited on top of metallic electrodes at nearly ambient temperature. Therefore, suspended ultra-clean SWCNT weak links could also be created by combining the mechanical transfer schemes [11-16] and our post-annealing step. The need of only moderate annealing

Address correspondence to Jukka-Pekka Kaikkonen, jukka-pekka.kaikkonen@aalto.fi; Pertti J. Hakonen, pertti.hakonen@aalto.fi

Note: The original version of this article was revised because of retrospective Open Access. 
temperatures for making SWCNT weak links on MoRe/Pd extends the range of other materials that can be placed on the device chip. For example, ferromagnetic materials or conventional oxide tunnel junctions could be used in the devices. This extended palette of materials and circuit elements paves the way for realizations of more complex hybrid devices with clean superconducting SWCNTs.

\section{Experimental methods}

Our SWCNT devices were fabricated on a $525-\mu \mathrm{m}$-thick heavily p-doped silicon wafer with a 280 -nm-thick thermally grown silicon dioxide on top. The wafer was first coated with an 80-nm-thick 60-40 alloy of MoRe by sputtering from two targets simultaneously. The substrate holder of our sputter (DCA M4500; DCA Instruments) was heated up to $\sim 700{ }^{\circ} \mathrm{C}$ prior to MoRe coating of the wafer, and this temperature was maintained during the sputtering and $15 \mathrm{~min}$ beyond it. This wafer heating improved the mixing of MoRe alloy increasing the superconducting critical temperature $\left(T_{\mathrm{c}}\right)$ of MoRe. Electrode patterning was done in two steps. First, a set of alignment markers and 119 pairs of bonding pads connected with $10-\mu \mathrm{m}$ wide strip lines were patterned using photolithography. MoRe was etched away with $\mathrm{SF}_{6} / \mathrm{Ar}$ reactive ion etching (RIE) in an Oxford Plasmalab 80 Plus system with a patterned 1.4- $\mu$ m-thick AZ 5214 photoresist acting as the etching mask. A short oxygen plasma ashing was done after the MoRe etch with the intent to clean fluorocarbon polymers formed during the $\mathrm{SF}_{6} / \mathrm{Ar}$ etching. In the second lithography step, the sourcedrain gaps were first patterned on a 500 -nm-thick polymethyl methacrylate (PMMA) film using standard electron beam lithography (EBL). Metallization was removed with RIE etching in the same way as in the first step but etching was continued deeper into $\mathrm{SiO}_{2}$ to guarantee suspension of the deposited SWCNTs. PMMA is not very resistant to RIE etching so the source-drain gap patterned with EBL became wider after the etching. Therefore, 150 -nm-wide lines were patterned with EBL to reach 300- to 350-nm-wide trenches on MoRe after the etching. Finally, the chips were carefully cleaned by sonicating them in acetone. A schematic illustration of the electrode fabrication procedure is presented in Fig. S1(a) in the Electronic Supplementary Material (ESM). The device layout is illustrated in Fig. 1(a).

An additional lithography step was done for the samples with a Pd contact layer. These steps are illustrated in Fig. S1(b) in the ESM. First, two $10 \mu \mathrm{m} \times 10 \mu \mathrm{m}$ windows next to the source-drain gap were patterned on PMMA using EBL. The exposed areas of MoRe were cleaned for $15 \mathrm{~s}$ with $\mathrm{SF}_{6} / \mathrm{Ar}$ plasma and the sample was immediately loaded into an electron beam evaporator. In the evaporator the sample was further cleaned with weak argon plasma for $1 \mathrm{~min}$ and subsequently, a 5-nm-thick layer of Pd was evaporated. The lift-off and final cleaning were done in a $50{ }^{\circ} \mathrm{C}$ acetone bath.

For SWCNT deposition the device chips were loaded into a purpose-built thermophoretic precipitator that was connected to the gas outlet of the FC-CVD reactor. SWCNTs were grown in the FC-CVD process with ferrocene as the catalyst precursor and carbon monoxide (CO) as the carbon feedstock. Additional carbon dioxide $\left(\mathrm{CO}_{2}\right)$ flow was used to control the mean diameter of the SWCNTs. Thermophoretic precipitator and the deposition process are described in Refs. [30, 31] and the FC-CVD process in Refs. [30, 32]. The setup used for the synthesis and the deposition of SWCNTs is schematically shown in Fig. 1(b).

To obtain the desired density and spatial uniformity of the deposited SWCNTs, the FC-CVD process parameters and deposition times were first calibrated by running test (a)

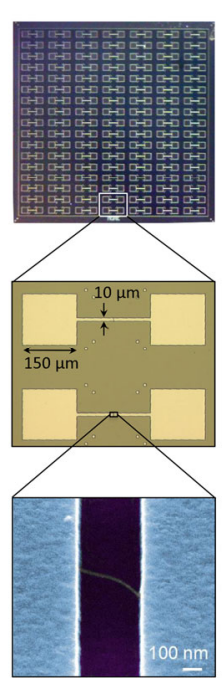

(b)

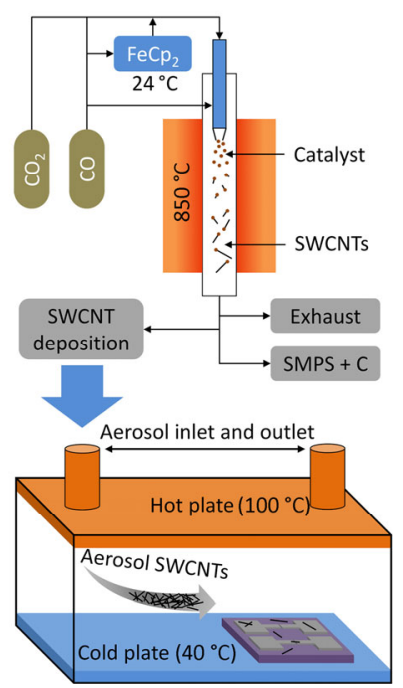

Figure 1 (a) Electrode layout of the $5 \mathrm{~mm} \times 5 \mathrm{~mm} \mathrm{Si} \mathrm{chip,} \mathrm{an} \mathrm{optical}$ image of two electrode pairs, and an SEM image of a suspended SWCNT (from top to bottom). (b) Schematic of the setup used for the synthesis and deposition of SWCNTs. Flow of CO is passed through a cartridge containing ferrocene $\left(\mathrm{FeCp}_{2}\right)$ and the resulting ferrocene-containing gas is directed into the CVD reactor through a water-cooled injector probe. The growth of SWCNTs takes place in the CVD reactor and the outcoming gas flow carries the SWCNTs into the thermophoretic precipitator. In the thermophoretic precipitator the gas flows between two plates that are kept at different temperatures and the SWCNTs are thermophoretically deposited onto the target chip. The gas phase number concentration is monitored using a scanning mobility particle sizer with a condensed particle counter (SMPS $+\mathrm{C}$ ).

depositions on bare $\mathrm{Si} / \mathrm{SiO}_{2}$ chips and imaging the resulting configurations on a scanning electron microscope (SEM). A typical SEM micrograph displaying deposited SWCNTs on a $\mathrm{Si} / \mathrm{SiO}_{2}$ chip is shown in Fig. S2 in the ESM. The SWCNT gas phase number concentration was measured using a scanning mobility particle sizer with a condensed particle counter (SMPS+C system; GRIMM Aerosol Technik GmbH, Germany). The number concentration was kept around $10^{5} \mathrm{~cm}^{-3}$ to prevent unnecessary bundling of the tubes [32] (see Fig. S3(a) in the ESM) and to allow for better control of their density on the target chip. Mean diameter of the SWCNTs was determined by ultraviolet/visible/near-infrared (UV/vis/NIR) absorbance spectroscopy (PerkinElmer LAMBDA 950 UV/vis/NIR spectrophotometer; PerkinElmer Inc.) of separately collected thin-film samples [30] (see Fig. S3(b) in the ESM). Typical average diameter of the SWCNTs was $1.8 \mathrm{~nm}$ with $0.3 \mathrm{~nm}$ standard deviation.

The SWCNT deposition took place in the thermophoretic precipitator where the SWCNT-carrying aerosol flowed between two plates that were kept at different temperatures. The bottom plate was kept at ambient temperature by water cooling and the top plate was heated to $100{ }^{\circ} \mathrm{C}$ by two power resistors [30]. During the thermophoretic deposition process SWCNTs landed randomly on the target chip located on the water-cooled bottom plate, and some SWCNTs became suspended across the prepatterned source-drain electrodes.

After the SWCNT deposition step, the samples were loaded into an infrared rapid thermal annealing oven (MILA-5000; ADVANCE RIKO, Inc., Yokohama, Japan) in order to improve electrical conductivity of the contacts between the suspended SWCNTs and the electrodes. The oven was flushed with $\mathrm{Ar} / \mathrm{H}_{2}$ mixture $\left(3 \%\right.$ of $\left.\mathrm{H}_{2}\right)$ and pumped for 15-30 min using a turbo pump before the annealing. The residual pressure was about $3 \times 10^{-4}$ mbar. Samples with MoRe electrodes were heated up to $900{ }^{\circ} \mathrm{C}$ in $90 \mathrm{~s}$ and annealed at this temperature for $5 \mathrm{~min}$. 
$\mathrm{MoRe} / \mathrm{Pd}$ samples were heated up to $220^{\circ} \mathrm{C}$ in $60 \mathrm{~s}$ and annealed for $10 \mathrm{~min}$.

The devices were first characterized at ambient conditions using a semiautomatic probe station (PA150; Süss MicroTec AG) connected to a semiconductor parameter analyzer (Agilent 4156B). More accurate gate sweeps were then carried out for the low resistance samples in a vacuum probe station. Devices that showed quasi-metallic behavior and minimum resistance below $20 \mathrm{k} \Omega$ within the gate voltage range of $\pm 10 \mathrm{~V}$ were attached and bonded into a sample holder, and loaded into a dilution refrigerator (BF-LD250, Bluefors Cryogenics) for low temperature characterization.

Low temperature measurements were performed in a quasifour-terminal configuration using standard lock-in techniques (see Section S4 in the ESM). Source and drain signals were filtered by 3 -stage RC filters and 1.5-m-long Thermocoax cables at the base temperature. Twisted pair lines served as the connection between cryogenic and room-temperature circuits. Heavily p-doped Si chip worked as the gate electrode and its connection from the mixing chamber temperature to $300 \mathrm{~K}$ was made using a 3-m-long Thermocoax cable. Attenuated coaxial cables were also connected to the source and gate lines via bias tees to facilitate high frequency driving of the sample. Large bias resistors (115 M $\Omega$ for DC and $1 \mathrm{G} \Omega$ for low frequency $\mathrm{AC}$ ) were employed at room temperature in the current bias measurements, while low-impedance voltage dividers were used at their place in voltage bias measurements.

\section{Results}

Figures 2(a) and 2(b) illustrate initial semiautomatic probe station measurements of a MoRe chip after the SWCNT deposition and annealing processes. This particular chip showed 16 SWCNTs connecting the source-drain electrodes with 13 of them being metallic or small band gap SWCNTs. Only a small fraction of the 119 source-drain pairs had thus been contacted with a SWCNT. This low density of SWCNTs was, however, desirable in order to avoid several SWCNTs bridging over a single trench. A couple of the devices on this chip had a minimum resistance below $20 \mathrm{k} \Omega$ and these were considered for further characterization. Thus, the number of low resistance devices per source-drain pair was rather low
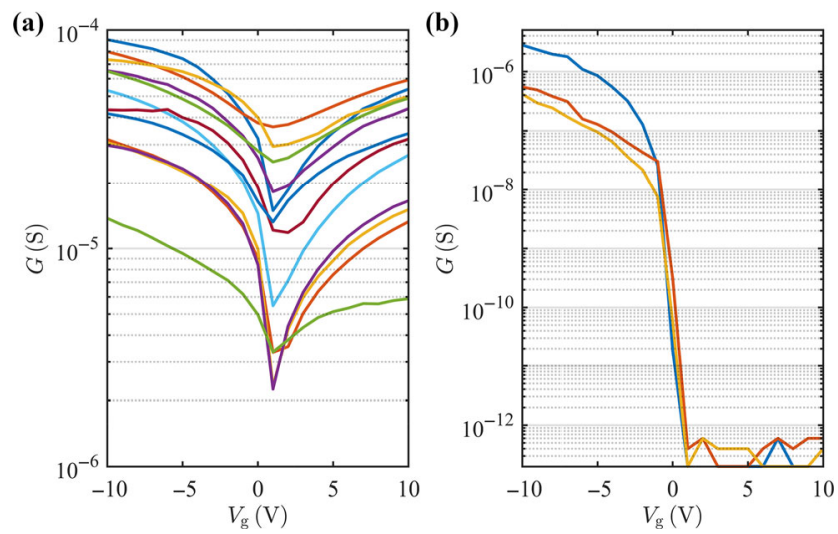

Figure 2 Initial characterization of a MoRe chip after the FC-CVD and annealing processes; data measured on a semiautomatic probe station. (a) Conductance $(G)$ versus gate voltage $\left(V_{\mathrm{g}}\right)$ of metallic and small band gap SWCNTs. Thirteen devices showed this kind of quasi-metallic transport behavior on this chip. One of them had a significantly lower conductance than the others and is not included in the figure. (b) Conductance versus gate voltage of large band gap semiconducting SWCNTs. Three semiconducting SWCNTs were found on this chip. Other source-drain pairs had no SWCNTs contacting them. but every chip typically had a few of them once the FC-CVD process parameters were properly adjusted.

Figure 3(a) plots the differential conductance of a SWCNT device on MoRe electrodes versus bias voltage $\left(V_{\mathrm{b}}\right)$ and gate voltage $\left(V_{\mathrm{g}}\right)$ at $T \approx 7.2 \mathrm{~K}$. At this temperature, the electrodes were still in the normal conducting state since the superconducting transition of the electrodes was observed as a resistance drop at $T_{\mathrm{c}} \approx 6.5 \mathrm{~K}$. Fabry-Pérot interference pattern with high conductance is clearly seen in this figure, indicating that the device is in the so-called open quantum dot regime. Fourier transform of the Fabry-Pérot pattern is shown in Fig. S6(b) in the ESM. Two Fabry-Pérot "diamonds" are also illustrated in Fig. 3(a) with solid and dashed lines. Source-drain voltage at the top corner of the diamond $\left(V_{\text {sd }}\right)$ can be used to extract the energy level spacing $\Delta E \approx 4.6 \mathrm{meV}$. This energy level spacing corresponds to longitudinal quantization of $L=$ $h v_{\mathrm{F}} / 2 \Delta E=360 \mathrm{~nm}$ [33], where $v_{\mathrm{F}} \approx 8 \times 10^{5} \mathrm{~m} \cdot \mathrm{s}^{-1}$ is the Fermi velocity and $h$ the Planck constant. The extracted quantization length is in good agreement with the source-drain separation of $330 \mathrm{~nm}$, which was measured afterwards on an SEM (see Fig. S7(a) in the ESM). The observation of Fabry-Pérot resonances demonstrates that transparent contacts can be obtained by post-annealing SWCNTs that have been deposited on MoRe electrodes at nearly ambient conditions.

The presence of proximity-induced supercurrents in the sample was verified by current biased measurements. Figure 3(b) shows a $V-I$ curve obtained from such a measurement. $V-I$ characteristic displays strong hysteresis with a sharp transition from supercurrent to resistive branch at the switching current $I_{\mathrm{sw}} \approx 53 \mathrm{nA}$ and a reverse transition at much lower retrapping current $I_{\mathrm{r}} \approx 5 \mathrm{nA}$. In SNS weak links the junction capacitance $\left(C_{j}\right)$, other circuit capacitances $[34,35]$, or an increase of electron temperature [36] can be responsible for hysteresis but making accurate estimates of these factors is difficult.

In the resistively and capacitively shunted junction (RCSJ) model hysteresis is described by the Stewart-McCumber

(a)

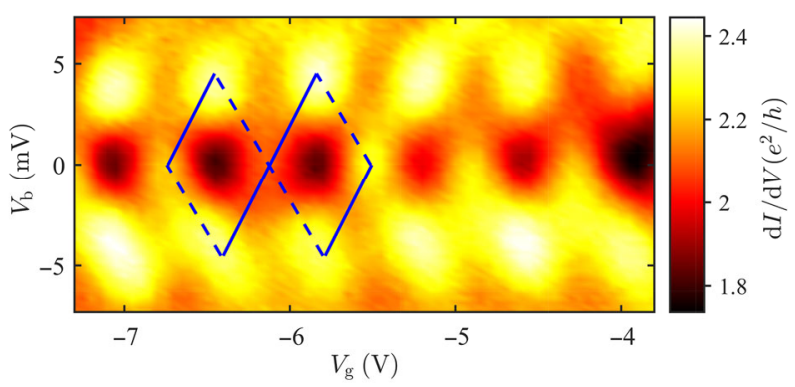

(b)
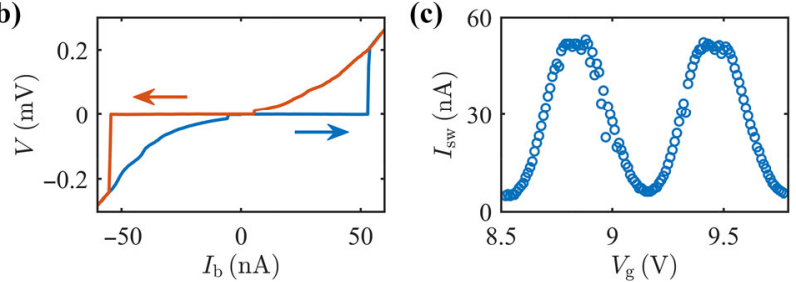

Figure 3 (a) Differential conductance versus bias voltage $\left(V_{\mathrm{b}}\right)$ and gate voltage $\left(V_{\mathrm{g}}\right)$ above $T_{\mathrm{c}}$ at $T \approx 7.2 \mathrm{~K}$. (b) $V-I$ curve measured with quasifour-terminal current bias configuration at $T \approx 10 \mathrm{mK}$. Gate voltage was $V_{\mathrm{g}}=9.45 \mathrm{~V}$. The transition from supercurrent to resistive branch occurs at $53 \mathrm{nA}$ and the reverse transition from resistive to the supercurrent branch at $5 \mathrm{nA}$. (c) Gate voltage modulation of the measured switching current. At each $V_{\mathrm{g}}$ only one $V-I$ curve was measured, from which the switching current $I_{\mathrm{sw}}$ was deduced. Measured $V_{\mathrm{g}}$ modulation of $I_{\mathrm{sw}}$ is not entirely smooth since the width of $I_{\mathrm{sw}}$ distribution makes scatter on the values obtained from a single measurement. 
parameter $\beta_{\mathrm{c}}=\omega_{\mathrm{p}}{ }^{2} R_{\mathrm{j}}{ }^{2} C_{\mathrm{j}}^{2}$, where $\omega_{\mathrm{p}}=\left(2 e I_{\mathrm{c}} / \hbar C_{\mathrm{j}}\right)^{1 / 2}$ is the plasma frequency, $I_{\mathrm{c}}$ is the critical current, and $R_{\mathrm{j}}$ is the shunt resistance. At zero temperature, $\beta_{c}$ is related to the hysteresis by [37]

$$
\frac{I_{\mathrm{c}}}{I_{\mathrm{r}}}=\frac{\pi \sqrt{\beta_{\mathrm{c}}}}{4}
$$

where $I_{\mathrm{r}}$ is the retrapping current. From Eq. (1) we can then estimate $C_{\mathrm{j}}$ that would correspond to the observed hysteresis within the RCSJ model. Assuming that the critical current can be substituted by the switching current, $I_{\mathrm{c}} \approx I_{\mathrm{sw}}$, and shunt resistance is equal to the normal state resistance $\left(R_{\mathrm{N}}\right), R_{\mathrm{j}} \approx$ $R_{\mathrm{N}} \approx 10 \mathrm{k} \Omega$, we obtain $\beta_{\mathrm{c}} \approx 180$ and $C_{\mathrm{j}} \approx 11 \mathrm{fF}$. The obtained capacitance is rather large for the effective capacitance of the QD, indicating that other circuit capacitances or electron heating contributes to the observed hysteresis.

The gate voltage modulation of $I_{\mathrm{sw}}$ is depicted in Fig. 3(c). The gate voltage range in this figure is different from Fig. 3(a) but the $I_{\mathrm{sw}}$ modulation follows nearly same gate period as the conductance oscillations in Fig. 3(a). Therefore, this $I_{\mathrm{sw}}$ modulation is related to the gate tuning of the SWCNT QD energy levels with respect to the Fermi energy of the leads. Switching current, however, exhibits rather low minimum value and thus, $I_{\text {sw }}$ oscillations have much larger relative amplitude than $R_{\mathrm{N}}$ oscillations of the SWCNT QD. This suppression of $I_{\mathrm{sw}}$ has been previously seen in SWCNT weak links [34] and it has been explained by the influence of the electromagnetic environment, which yields to $I_{\mathrm{sw}} \propto I_{c}^{3 / 2}$ dependence of $I_{\mathrm{sw}}[34,38]$.

Proximity-induced supercurrents were also measured from a sample that had a Pd contact layer on top of MoRe. Figure 4 shows two $V-I$ curves measured from such a MoRe/Pd device using current bias. A sharp transition from the supercurrent to the resistive branch is observed also in this sample, and the largest measured switching current was about $350 \mathrm{pA}$. However, SEM micrographs taken from this device after the measurements revealed that there were two SWCNTs suspended across the source-drain electrodes (see Fig. S7(b) in the ESM). Therefore, critical current through a single SWCNT might have been even below the measured value. Normal state resistance of this sample was $R_{\mathrm{N}} \approx 20 \mathrm{k} \Omega$. The resistance is large compared to the MoRe device presented above, but it can only partially account for the low switching current of this sample (see below).

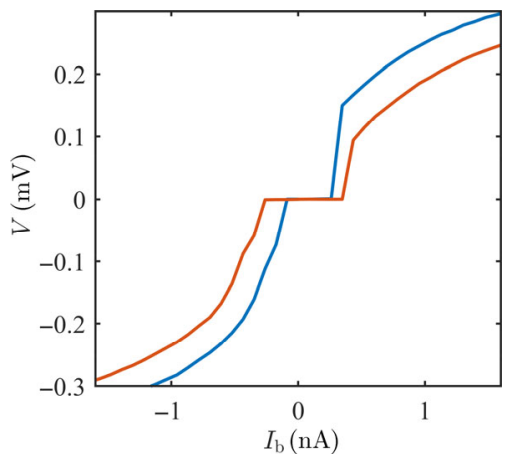

Figure 4 Bias current $\left(I_{\mathrm{b}}\right)$ versus source-drain voltage of the $\mathrm{MoRe} / \mathrm{Pd}$ sample measured at $V_{\mathrm{g}}=-3.71 \mathrm{~V}$ (blue) and $V_{\mathrm{g}}=-3.72 \mathrm{~V}$ (red). The bias current was swept from negative to positive values. The maximum switching current is about $350 \mathrm{pA}$.

\section{Discussion}

Our results unambiguously show that proximity supercurrents can be induced into aerosol-synthesized SWCNTs deposited on top of the electrodes at nearly ambient conditions and annealed afterwards to achieve transparent contacts. SWCNTs on MoRe contacts were found to support larger supercurrents than SWCNTs on MoRe/Pd contacts. SWCNTs on MoRe typically showed resistances above $100 \mathrm{k} \Omega$ right after the deposition and to reach transparent contacts, these samples were annealed at $900{ }^{\circ} \mathrm{C}$, which is comparable to the temperature used in the CVD growth of SWCNTs. The effect of annealing is illustrated in Fig. S8 in the ESM, which compares the on-state conductance of the devices before and after annealing at $900{ }^{\circ} \mathrm{C}$. We believe that, to generate transparent contacts to MoRe, high annealing temperature is needed mainly to decompose Mo oxides and to form Mo carbides. In addition, high temperature annealing may improve contact transparencies by increasing the contact area [39]. Composition of co-sputtered MoRe film has been previously studied by X-ray photoelectron spectroscopy (XPS) in Ref. [40] and higher Mo concentration was found on the surface of the film due formation of molybdenum oxides, namely $\mathrm{MoO}_{3}$ and $\mathrm{MoO}_{2}$. Reduction of these oxides was seen after a CVD process where the film was exposed to methane-hydrogen atmosphere at $800{ }^{\circ} \mathrm{C}$ for several minutes. The alloy composition of our film differed from that in Ref. [40], but it is very likely that similar oxides were formed on the surface of our MoRe films once exposed to air and accordingly, these oxides were decomposed during the employed high temperature annealing.

Molybdenum deposited on top of SWCNTs has been shown to form $\mathrm{Mo}_{2} \mathrm{C}$ carbide above $\sim 800{ }^{\circ} \mathrm{C}$, creating an abrupt but transparent end-bonded contact to SWCNTs [41]. The same carbide formation process presumably played a role in creating transparent contacts to SWCNTs also in our devices, since we found significant decrease in the device resistances when annealed at temperatures above $800{ }^{\circ} \mathrm{C}$.

The above considerations suggest that the high temperature annealing is necessary in order to produce good electrical contacts between MoRe leads and a SWCNT. Therefore, such samples still need to be exposed to equally high temperatures as in the in situ CVD growth of SWCNTs. When SWCNTs are grown directly over the electrodes, the high temperature atmosphere always contains hydrogen and carbon. Our fabrication method has no such constraints, and the annealing can thus be done in vacuum or in an inert atmosphere. This possibility will be beneficial for high frequency devices as the XPS measurements have shown that a significant concentration of carbon is introduced into MoRe films during the CVD process [40]. Consequently, the CVD process increases the resistivity of MoRe films and reduces their superconducting transition temperature. Quality factor of microwave resonators fabricated from the film decreases in the CVD process as well [40, 42, 43]. We performed annealing in vacuum at a chamber pressure of $p \approx 3 \times 10^{-4}$ mbar after flushing with an $\mathrm{Ar} / \mathrm{H}_{2}$ gas mixture. Due to the modest vacuum quality, some amounts of oxygen, carbon dioxide and hydrogen were still present in the annealing atmosphere. However, the film degradation due to oxygen, carbon or hydrogen exposure at high temperature can be avoided in our fabrication scheme by improving the vacuum quality.

Palladium contact layer significantly reduced the annealing temperature needed to form transparent contacts (see Fig. S9 in the ESM) and to induce proximity supercurrents into SWCNTs. This widely extends the range of materials and devices that can be placed on the same chip with a superconducting SWCNT weak link and consequently, provides new possibilities for hybrid circuit experiments with ultra-clean SWCNT weak links. For example, commonly used aluminium tunnel junctions have been shown to be stable to vacuum annealing up to $200^{\circ} \mathrm{C}$ [44], comparable to the annealing temperature we used for 
SWCNTs on MoRe/Pd. Common magnetic materials such as nickel-iron permalloy or cobalt can also withstand similar annealing temperatures in vacuum.

Magnetic properties of the Pd films may provide the reason for rather small supercurrents in our SWCNT devices on top of $\mathrm{MoRe} / \mathrm{Pd}$ electrodes. Bulk Pd in its crystalline form is paramagnetic but it has a high magnetic susceptibility and it is close to the phase transition to ferromagnetism. Consequently, just a small concentration of magnetic impurities can promote ferromagnetic ordering in Pd, and even lattice disorder [45-47], lattice expansion [48, 49], and reduced dimensionality [50-53] may be sufficient to induce magnetism at cryogenic temperatures. This suggests that the Pd deposition needs to be done with great care to avoid magnetic ordering in the Pd film. We did not characterize the magnetic properties of our $5-\mathrm{nm}$ thick Pd films so we cannot conclude for certain that this effect caused the reduced supercurrents in our sample.

The strength of the proximity effect in Pd might also have been weakened by the presence of an oxide layer between MoRe and Pd. Our MoRe film was exposed to air before the evaporation of the Pd layer and it is unclear whether the surface oxides were fully removed by the weak argon plasma cleaning performed in the evaporator prior to the Pd deposition. Depositing MoRe and Pd in the same vacuum chamber could thus increase critical currents while simultaneously simplify the fabrication process. Finally, we only tested 5-nm-thick Pd layers and annealing at $220^{\circ} \mathrm{C}$ so optimization of these parameters should enable larger supercurrents. For example, the evaporated $\mathrm{Pd} / \mathrm{Al}$ contacts on SWCNTs in Ref. [54] displayed a supercurrent of $\approx 6 \mathrm{nA}$ through a 7-nm-thick Pd contact layer.

\section{Conclusions}

In summary, we have presented a new scheme for fabrication of individual suspended SWCNT weak links in which aerosolsynthesized pristine SWCNTs are directly deposited onto prefabricated electrodes at nearly ambient temperature and later post-annealed in vacuum to generate good electrical contacts between the electrodes and the SWCNTs. The fabricated SWCNTs are not exposed to an electron beam or microfabrication chemicals during the process. Samples were fabricated both on MoRe and MoRe/Pd electrodes and annealed in vacuum at 900 and $220{ }^{\circ} \mathrm{C}$, respectively. Proximityinduced supercurrents were demonstrated by measuring switching currents using a quasi-four-terminal current bias configuration. The largest switching currents measured from our SWCNTs on MoRe electrodes were $53 \mathrm{nA}$, over four times larger than previously reported values for clean suspended SWCNT weak links [18]. SWCNTs on MoRe/Pd electrodes instead showed switching currents up to $350 \mathrm{pA}$. The low annealing temperature necessary to induce proximity supercurrents into SWCNTs on MoRe/Pd contacts extends the range of materials and device configurations that can be employed together with clean SWCNT weak links on a single chip.

\section{Acknowledgements}

The authors thank Unto Suominen from VTT Technical Research Centre of Finland for help with the MoRe sputtering and Pasi Häkkinen for assistance with the fabrication at the initial stage of this project. This work was supported by the Academy of Finland projects 314448 (BOLOSE) and 312295 (CoE, Quantum Technology Finland) as well as by ERC (grant no. 670743). The research also received partial funding from the European Union Seventh Framework Program FP7 Nanosciences,
Nanotechnologies, Materials and new Production Technologies (FP7/2007-2013) under Grant Agreement No. 604472 (IRENA project) and the Aalto Energy Efficiency (AEF) Research Program through the MOPPI project. In addition, the research was partially supported by the Academy of Finland (Luonnontieteiden ja Tekniikan Tutkimuksen Toimikunta) via projects 286546 (DEMEC) and 292600 (SUPER), as well as by TEKES Finland via projects 3303/31/2015 (CNT-PV) and 1882/31/ 2016 (FEDOC). This research project utilized the Aalto University OtaNano/ NanoFab and Aalto-NMC facilities, and Low Temperature Laboratory infrastructure, which is part of European Microkelvin Platform. J.-P. K. is grateful for the financial support from Vilho, Yrjö and Kalle Väisalä Foundation of the Finnish Academy of Science and Letters.

Funding note: Open access funding provided by Aalto University.

Electronic Supplementary Material: Supplementary material (SEM micrographs, SMPS+C data, UV/vis/NIR optical absorption spectrum, quasi-four-terminal configuration, Fourier transform of the Fabry-Pérot pattern, contact annealing data) is available in the online version of this article at https://doi.org/10.1007/ s12274-020-3032-1.

Open Access This article is licensed under a Creative Commons Attribution 4.0 International License, which permits use, sharing, adaptation, distribution and reproduction in any medium or format, as long as you give appropriate credit to the original author(s) and the source, provide a link to the Creative Commons licence, and indicate if changes were made.

The images or other third party material in this article are included in the article's Creative Commons licence, unless indicated otherwise in a credit line to the material. If material is not included in the article's Creative Commons licence and your intended use is not permitted by statutory regulation or exceeds the permitted use, you will need to obtain permission directly from the copyright holder.

To view a copy of this licence, visit http://creativecommons.org/licenses/by/4.0/.

\section{References}

[1] Laird, E. A.; Kuemmeth, F.; Steele, G. A.; Grove-Rasmussen, K.; Nygård, J.; Flensberg, K.; Kouwenhoven, L. P. Quantum transport in carbon nanotubes. Rev. Mod. Phys. 2015, 87, 703-764.

[2] Sapmaz, S.; Jarillo-Herrero, P.; Blanter, Y. M.; Dekker, C.; Van Der Zant, H. S. J. Tunneling in suspended carbon nanotubes assisted by longitudinal phonons. Phys. Rev. Lett. 2006, 96, 026801.

[3] Steele, G. A.; Hüttel, A. K.; Witkamp, B.; Poot, M.; Meerwaldt, H. B.; Kouwenhoven, L. P.; Van Der Zant, H. S. J. Strong coupling between single-electron tunneling and nanomechanical motion. Science 2009, 325, 1103-1107.

[4] Leturcq, R.; Stampfer, C.; Inderbitzin, K.; Durrer, L.; Hierold, C.; Mariani, E.; Schultz, M. G.; Von Oppen, F.; Ensslin, K. FranckCondon blockade in suspended carbon nanotube quantum dots. Nat. Phys. 2009, 5, 327-331.

[5] Benyamini, A.; Hamo, A.; Kusminskiy, S. V.; Von Oppen, F.; Ilani, S. Real-space tailoring of the electron-phonon coupling in ultraclean nanotube mechanical resonators. Nat. Phys. 2014, 10, 151-156.

[6] Chaste, J.; Eichler, A.; Moser, J.; Ceballos, G.; Rurali, R.; Bachtold, A. A nanomechanical mass sensor with yoctogram resolution. Nat. Nanotechnol. 2012, 7, 301-304.

[7] Häkkinen, P.; Isacsson, A.; Savin, A.; Sulkko, J.; Hakonen, P. Charge sensitivity enhancement via mechanical oscillation in suspended carbon nanotube devices. Nano Lett. 2015, 15, 1667-1672.

[8] Moser, J.; Güttinger, J.; Eichler, A.; Esplandiu, M. J.; Liu, D. E.; Dykman, M. I.; Bachtold, A. Ultrasensitive force detection with a nanotube mechanical resonator. Nat. Nanotechnol. 2013, 8, 493-496. 
[9] Ganzhorn, M.; Klyatskaya, S.; Ruben, M.; Wernsdorfer, W. Carbon nanotube nanoelectromechanical systems as magnetometers for single-molecule magnets. ACS Nano 2013, 7, 6225-6236.

[10] Cao, J.; Wang, Q.; Dai, H. J. Electron transport in very clean, asgrown suspended carbon nanotubes. Nat. Mater. 2005, 4, 745-749.

[11] Wu, C. C.; Liu, C. H.; Zhong, Z. H. One-step direct transfer of pristine single-walled carbon nanotubes for functional nanoelectronics. Nano Lett. 2010, 10, 1032-1036.

[12] Waissman, J.; Honig, M.; Pecker, S.; Benyamini, A.; Hamo, A.; Ilani, $\mathrm{S}$. Realization of pristine and locally tunable one-dimensional electron systems in carbon nanotubes. Nat. Nanotechnol. 2013, 8, 569-574.

[13] Muoth, M.; Hierold, C. Transfer of carbon nanotubes onto microactuators for hysteresis-free transistors at low thermal budget. In Proceedings of 2012 IEEE 25th International Conference on Micro Electro Mechanical Systems (MEMS), Paris, France, 2012, pp 1352-1355.

[14] Gramich, J.; Baumgartner, A.; Muoth, M.; Hierold, C.; Schönenberger, C. Fork stamping of pristine carbon nanotubes onto ferromagnetic contacts for spin-valve devices. Phys. Status Solidi B 2015, 252, 2496-2502.

[15] Blien, S.; Steger, P.; Albang, A.; Paradiso, N.; Hüttel, A. K. Quartz tuning-fork based carbon nanotube transfer into quantum device geometries. Phys. Status Solidi B 2018, 255, 1800118.

[16] Pei, F.; Laird, E. A.; Steele, G. A.; Kouwenhoven, L. P. Valley-spin blockade and spin resonance in carbon nanotubes. Nat. Nanotechnol. 2012, 7, 630-634

[17] Keijzers, C. J. H. Josephson effects in carbon nanotube mechanical resonators and graphene. Ph.D. Dissertation, Delft University of Technology, Delft, 2012.

[18] Schneider, B. H.; Etaki, S.; Van Der Zant, H. S. J.; Steele, G. A. Coupling carbon nanotube mechanics to a superconducting circuit. Sci. Rep. 2012, 2, 599.

[19] Lim, J. S.; López, R.; Aguado, R. Josephson current in carbon nanotubes with spin-orbit interaction. Phys. Rev. Lett. 2011, 107, 196801.

[20] Cleuziou, J. P.; Wernsdorfer, W.; Bouchiat, V.; Ondarçuhu, T.; Monthioux, M. Carbon nanotube superconducting quantum interference device. Nat. Nanotechnol. 2006, 1, 53-59.

[21] Cleuziou, J. P.; Wernsdorfer, W.; Bouchiat, V.; Ondarçuhu, T.; Monthioux, M. Carbon nanotube based magnetic flux detector for molecular spintronics. Phys. Status Solidi B 2007, 244, 4351-4355.

[22] Bouchiat, V. Detection of magnetic moments using a nano-SQUID: Limits of resolution and sensitivity in near-field SQUID magnetometry. Supercond. Sci. Technol. 2009, 22, 064002.

[23] Braunecker, B.; Burset, P.; Yeyati, A. L. Entanglement detection from conductance measurements in carbon nanotube cooper pair splitters. Phys. Rev. Lett. 2013, 111, 136806.

[24] Hels, M. C.; Braunecker, B.; Grove-Rasmussen, K.; Nygård, J. Noncollinear spin-orbit magnetic fields in a carbon nanotube double quantum dot. Phys. Rev. Lett. 2016, 117, 276802.

[25] Bouchiat, V.; Chtchelkatchev, N.; Feinberg, D.; Lesovik, G. B.; Martin, T.; Torrès, J. Single-walled carbon nanotube-superconductor entangler: Noise correlations and Einstein-Podolsky-Rosen states. Nanotechnology 2003, 14, 77-85.

[26] Padurariu, C.; Keijzers, C. J. H.; Nazarov, Y. V. Effect of mechanical resonance on josephson dynamics. Phys. Rev. B 2012, 86, 155448.

[27] Khosla, K. E.; Vanner, M. R.; Ares, N.; Laird, E. A. Displacemon electromechanics: How to detect quantum interference in a nanomechanical resonator. Phys. Rev. X 2018, 8, 021052.

[28] Lechner, L.; Gaaß, M.; Paila, A.; Sillanpää, M. A.; Strunk, C.; Hakonen, P. J. Microwave reflection measurement of critical currents in a nanotube josephson transistor with a resistive environment. Nanotechnology 2011, 22, 125203.

[29] Ares, N.; Pei, T.; Mavalankar, A.; Mergenthaler, M.; Warner, J. H.; Briggs, G. A. D.; Laird, E. A. Resonant optomechanics with a vibrating carbon nanotube and a radio-frequency cavity. Phys. Rev. Lett. $\mathbf{2 0 1 6}$ 117, 170801.

[30] Laiho, P.; Mustonen, K.; Ohno, Y.; Maruyama, S.; Kauppinen, E. I. Dry and direct deposition of aerosol-synthesized single-walled carbon nanotubes by thermophoresis. ACS Appl. Mater. Interfaces 2017, 9, 20738-20747.

[31] Wei, N.; Laiho, P.; Khan, A. T.; Hussain, A.; Lyuleeva, A.; Ahmed, S.; Zhang, Q.; Liao, Y. P.; Tian, Y.; Ding, E. X. et al. Fast and ultraclean approach for measuring the transport properties of carbon nanotubes. Adv. Funct. Mater. 2020, 30, 1907150.

[32] Mustonen, K.; Laiho, P.; Kaskela, A.; Zhu, Z.; Reynaud, O.; Houbenov, N.; Tian, Y.; Susi, T.; Jiang, H.; Nasibulin, A. G. et al. Gas phase synthesis of non-bundled, small diameter single-walled carbon nanotubes with near-armchair chiralities. Appl. Phys. Lett. 2015, 107, 013106

[33] Liang, W. J.; Bockrath, M.; Bozovic, D.; Hafner, J. H.; Tinkham, M.; Park, H. Fabry-Perot interference in a nanotube electron waveguide. Nature 2001, 411, 665-669.

[34] Jarillo-Herrero, P.; Van Dam, J. A.; Kouwenhoven, L. P. Quantum supercurrent transistors in carbon nanotubes. Nature 2006, 439, 953-956.

[35] Tsuneta, T.; Lechner, L.; Hakonen, P. J. Gate-controlled superconductivity in a diffusive multiwalled carbon nanotube. Phys. Rev. Lett. 2007, 98, 087002.

[36] Courtois, H.; Meschke, M.; Peltonen, J. T.; Pekola, J. P. Origin of hysteresis in a proximity Josephson junction. Phys. Rev. Lett. 2008, 101, 067002 .

[37] Tinkham, M. Introduction to Superconductivity; 2th ed. McGrawHill: New York, 1996.

[38] Joyez, P.; Lafarge, P.; Filipe, A.; Esteve, D.; Devoret, M. H. Observation of parity-induced suppression of Josephson tunneling in the superconducting single electron transistor. Phys. Rev. Lett. 1994, 72, 2458.

[39] Roschier, L.; Tarkiainen, R.; Ahlskog, M.; Paalanen, M.; Hakonen, P. Multiwalled carbon nanotubes as ultrasensitive electrometers. Appl. Phys. Lett. 2001, 78, 3295-3297.

[40] Götz, K. J. G.; Blien, S.; Stiller, P. L.; Vavra, O.; Mayer, T.; Huber, T.; Meier, T. N. G.; Kronseder, M.; Strunk, C.; Hüttel, A. K. Co-sputtered MoRe thin films for carbon nanotube growth-compatible superconducting coplanar resonators. Nanotechnology 2016, 27, 135202.

[41] Cao, Q.; Han, S. J.; Tersoff, J.; Franklin, A. D.; Zhu, Y.; Zhang, Z.; Tulevski, G. S.; Tang, J. S.; Haensch, W. End-bonded contacts for carbon nanotube transistors with low, size-independent resistance. Science 2015, 350, 68-72.

[42] Singh, V.; Schneider, B. H.; Bosman, S. J.; Merkx, E. P. J.; Steele, G. A. Molybdenum-rhenium alloy based high-Q superconducting microwave resonators. Appl. Phys. Lett. 2014, 105, 222601.

[43] Blien, S.; Götz, K. J. G.; Stiller, P. L.; Mayer, T.; Huber, T.; Vavra, O.; Hüttel, A. K. Towards carbon nanotube growth into superconducting microwave resonator geometries. Phys. Status Solidi B 2016, 253, 2385-2390.

[44] Pop, I. M.; Fournier, T.; Crozes, T.; Lecocq, F.; Matei, I.; Pannetier, B.; Buisson, O.; Guichard, W. Fabrication of stable and reproducible submicron tunnel junctions. J. Vac. Sci. Technol. B 2012, 30, 010607.

[45] Takano, N.; Kai, T.; Shiiki, K.; Terasaki, F. Effect of copious vacancies on magnetims of Pd. Solid State Commun. 1996, 97, 153-156.

[46] Alexandre, S. S.; Anglada, E.; Soler, J. M.; Yndurain, F. Magnetism of two-dimensional defects in Pd: Stacking faults, twin boundaries, and surfaces. Phys. Rev. B 2006, 74, 054405.

[47] Rodríguez, I.; Valladares, R. M.; Hinojosa-Romero, D.; Valladares, A.; Valladares, A. A. Emergence of magnetism in bulk amorphous palladium. Phys. Rev. B 2019, 100, 024422.

[48] Moruzzi, V. L.; Marcus, P. M. Magnetism in fec rhodium and palladium. Phys. Rev. B 1989, 39, 471-474.

[49] Chen, H.; Brener, N. E.; Callaway, J. Electronic structure, optical and magnetic properties of fcc palladium. Phys. Rev. B 1989, 40 (3), 1443-1449.

[50] Sampedro, B.; Crespo, P.; Hernando, A.; Litrán, R.; López, J. C. S.; Cartes, C. L.; Fernandez, A.; Ramírez, J.; Calbet, J. G.; Vallet, M. Ferromagnetism in fcc twinned $2.4 \mathrm{~nm}$ size Pd nanoparticles. Phys. Rev. Lett. 2003, 91, 237203.

[51] Shinohara, T.; Sato, T.; Taniyama, T. Surface ferromagnetism of Pd fine particles. Phys. Rev. Lett. 2003, 91, 197201.

[52] Delin, A.; Tosatti, E.; Weht, R. Magnetism in atomic-size palladium contacts and nanowires. Phys. Rev. Lett. 2004, 92, 057201.

[53] Hong, S. C.; Lee, J. I.; Wu, R. Q. Ferromagnetism in Pd thin films induced by quantum well states. Phys. Rev. B 2007, 75, 172402.

[54] Cleuziou, J. P.; Wernsdorfer, W.; Andergassen, S.; Florens, S.; Bouchiat, V.; Ondarçuhu, T; Monthioux, M. Gate-tuned high frequency response of carbon nanotube Josephson junction. Phys. Rev. Lett. 2007, 99, 117001 